\title{
A 1-Local Asymptotic 13/9-Competitive Algorithm for Multicoloring Hexagonal Graphs
}

\author{
Yong Zhang • Francis Y.L. Chin • Hong Zhu
}

Received: 17 August 2007 / Accepted: 9 June 2008

(C) Springer Science+Business Media, LLC 2008

\begin{abstract}
In the frequency allocation problem, we are given a mobile telephone network, whose geographical coverage area is divided into cells, wherein phone calls are serviced by assigning frequencies to them so that no two calls emanating from the same or neighboring cells are assigned the same frequency. The problem is to use the frequencies efficiently, i.e., minimize the span of frequencies used. The frequency allocation problem can be regarded as a multicoloring problem on a weighted hexagonal graph. In this paper, we give a 1-local asymptotic 4/3-competitive distributed algorithm for multicoloring a triangle-free hexagonal graph, which is a special case of hexagonal graph. Based on this result, we then propose a 1-local asymptotic 13/9-competitive algorithm for multicoloring the (general-case) hexagonal graph, thereby improving the previous 1-local 3/2-competitive algorithm.
\end{abstract}

Keywords Online algorithm $\cdot$ Multicoloring $\cdot$ Hexagonal graphs

\footnotetext{
A preliminary version of this paper appeared in the Proceedings of the 13th Annual International Computing and Combinatorics Conference (COCOON 2007), LNCS 4598, pp. 526-536.

Y. Zhang research was supported by European Regional Development Fund (ERDF).

F.Y.L. Chin research was supported by Hong Kong RGC Grant HKU-7113/07E.

H. Zhu research was supported by National Natural Science Fund (grant \#60496321).

Y. Zhang $(\bowtie) \cdot$ F.Y.L. Chin

Department of Computer Science, The University of Hong Kong, Hong Kong, Hong Kong

e-mail: yzhang@cs.hku.hk

F.Y.L. Chin

e-mail: chin@cs.hku.hk
}

H. Zhu

Institute of Theoretical Computing, East China Normal University, Shanghai, China

e-mail: hzhu@sei.ecnu.edu.cn 


\section{Introduction}

Wireless communication based on Frequency Division Multiplexing (FDM) technology is widely used in the area of mobile computing today. In such FDM networks, a geographic area is divided into small cellular regions or cells, each containing one base station. Base stations communicate with each other via a high-speed wired network. Calls between any two clients (even within the same cell) must be established through base stations. When a call arrives, the nearest base station must allocate a frequency from the available spectrum to the call without causing any interference to other calls. In practice, when the same frequency is assigned to two different calls emanating from cells that are geographically close to each other, interference may occur which distorts the radio signals. To avoid interference, the temptation is to use many frequencies. However, spectrum is a scarce resource, so efficient utilization of the available spectrum is essential for FDM networks.

The frequency allocation problem, including the off-line version and online version, has been extensively studied $[1-4,6,9,10,12,13,15]$. The research on this problem is mainly focused on the cellular network, where cells are hexagonal regions as shown in Fig. 1. In the off-line problem (the calls to be serviced are known a priori), McDiarmid and Reed [12] have shown that minimizing the span of frequencies to satisfy all the call requests is NP-hard, they also proved that $4 / 3$ is the lower bound of approximation ratio. Further, two 4/3-approximation algorithms were given in [12] and [14] respectively.

For the online version, there are mainly three strategies, which have been well studied: the fixed allocation assignment (FAA) [11], the greedy algorithm (Greedy) [5] and the hybrid algorithm [4]. FAA partitions cells into independent sets which are each assigned a separate set of frequencies. It is easy to see that FAA is 3-competitive as cellular networks are 3-colorable [5]. Greedy assigns the minimum available number (frequency) to a new call so that the call does not interfere with calls of the same or adjacent cells. Caragiannis et al. [5] proved that the competitive ratio of Greedy in cellular network is at least 17/7 and at most 2.5. Chan et al. [3] closed the gap and proved that Greedy is 17/7-competitive. Furthermore, Chan et al. [4] gave a 2-competitive algorithm, say hybrid, which can be regarded as a combination of FAA and Greedy. Since the lower bound of competitive ratio of frequency allocation in cellular networks is also 2, hybrid is optimal.

The frequency allocation problem in cellular network can be abstracted to the problem of multicoloring a weighted hexagonal graph, in which each vertex has a positive weight which specifies how many different colors have to be assigned to the

Fig. 1 Example of a cellular network (with hexagonal cells)

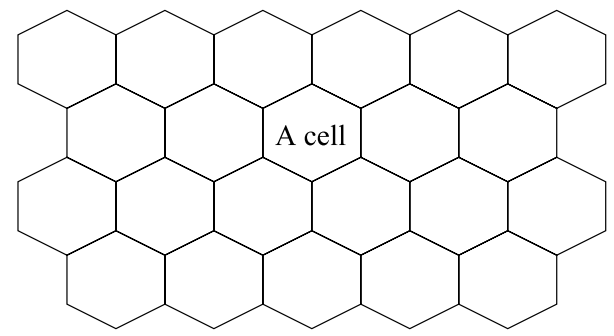


vertex. Given the constraint that the same color cannot be assigned to the same or adjacent vertices, the target is to minimize the number of assigned colors.

In frequency allocation problems, the size of cellular network is very large, when handling a call request, the computation will be very complex if all information of the whole network is needed. In reality, each server in a cell only knows its position before processing the request sequence of calls; when satisfying call requests, each server only knows its local information, i.e., some information within a fixed distance. Such kind of processing gives us a motivation to study distributed algorithms. In this paper, we focus on distributed algorithms for the multicoloring, i.e., each vertex is an independent server, which runs the algorithm to assign multicolors to the vertex based on what is known as $k$-local information. The concept of $k$-local distributed algorithms was introduced by Janssen et al. [8], where an algorithm is $k$-local if the computation at a vertex depends only on the information of the neighboring vertices of at most $k$ distance away (suppose each edge has unit distance). Similar to frequency allocation problem, we can assume that in multicoloring problem, each vertex also knows its position in the graph.

In [8], Janssen et al. proved (the next lemma) that a $k$-local $c$-approximate off-line algorithm can be easily converted to a $k$-local $c$-competitive online algorithm. Thus, to design a $k$-local online algorithm, we need only to focus on the $k$-local off-line problem.

Lemma 1 [8] Let A be a k-local c-approximate off-line algorithm for multicoloring. Then A can be converted into a k-local c-competitive online algorithm for multicoloring.

The problem of multicoloring a hexagonal graph is hard. But for some various graph classes, this problem may have a better performance. An interesting induced graph, triangle-free hexagonal graph, has been studied for the multicoloring problem $[7,16]$. A graph is triangle-free if there are no 3-cliques in the graph, i.e., there are no three mutually-adjacent vertices with positive weights. An example of a triangle-free hexagonal graph is shown in Fig. 2.

The best known competitive ratios for 0-, 1-, 2- and 4-local distributed algorithms for multicoloring on (general) hexagonal graphs are 3, 3/2, 4/3 and 4/3, respectively $[8,17]$. It is possible to do better for triangle-free hexagonal graphs. For

Fig. 2 An example of a triangle-free hexagonal graph, where solid circles are vertices with positive weights

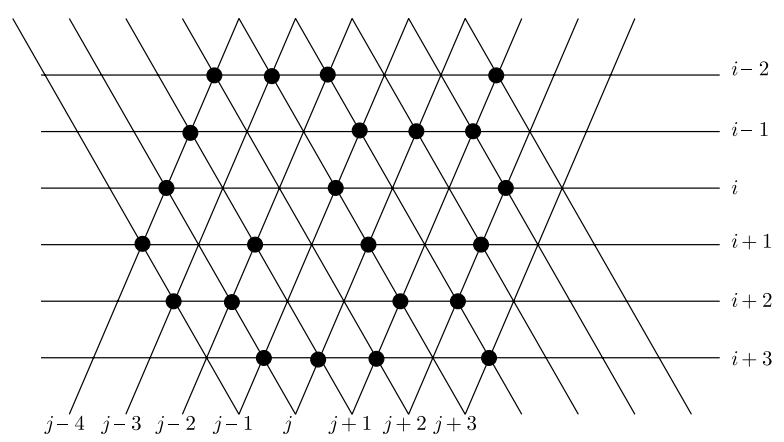


example, in [16], a 2-local 5/4 competitive algorithm was given, and an inductive proof for the 7/6 ratio was reported in [7].

The remaining of this paper is organized as follows. In Sect. 2, we introduce some preliminary terminology to be used in this paper. In Sect. 3, we give a 1-local asymptotic 4/3-competitive algorithm for multicoloring a triangle-free hexagonal graph. Based on this result, we then propose, in Sect. 4, a 1-local asymptotic 13/9-competitive algorithm for the multicoloring problem in hexagonal graph, which improves the previous 3/2-competitive result. Finally, we give the conclusion in Sect. 5.

\section{Preliminary Terminology}

Given a hexagonal graph with a non-negative weight at each vertex, we use a 2-coordinate system to represent each vertex. In particular, referring to the lines shown in Fig. 2, each vertex can be represented by coordinate $(i, j)$ where $i$ is the coordinate for the horizontal line, $j$ for the up-sloping line. For example, a vertex with coordinate $(i, j)$ and its six neighboring vertices, denoted by UL, L, DL, UR, R and DR, are represented as shown in Fig. 3.

Next, we define the parity of a vertex with respect to its various neighbors. We say that the parity of a vertex $v$ with coordinate $(i, j)$ is:

1. odd (alternatively, even) with respect to its $\mathrm{L}$ or $\mathrm{R}$ neighbor if $j \equiv 1 \bmod 2$ (correspondingly, $j \equiv 0 \bmod 2)$;

2. odd (alternatively, even) with respect to its UL or DR neighbor if $i \equiv 1 \bmod 2$ (correspondingly, $i \equiv 0 \bmod 2$ );

3. odd (alternatively, even) with respect to its DL or UR neighbor if $i \equiv 1 \bmod 2$ (correspondingly, $i \equiv 0 \bmod 2$ ).

Let $w_{v}$ be the weight of vertex $v$, which corresponds to the number of colors needed to multicolor $v$. After the multicoloring assignment, each vertex $v$ will be assigned a set $F_{v}$ of colors, such that $F_{v} \subset Z^{+}$, a set of positive integers, and $\left|F_{v}\right|=$ $w_{v}$, where, for any two adjacent vertices $u$ and $v, F_{u} \cap F_{v}=\phi$.

In order to help with this multicoloring assignment, we shall partition the set of vertices into three sets, each associated with a base color which denotes a separate set of colors (integers). Since a hexagonal graph is 3-colorable, we use three base colors,

Fig. 3 Coordinates of a vertex $(i, j)$ and its neighboring vertices

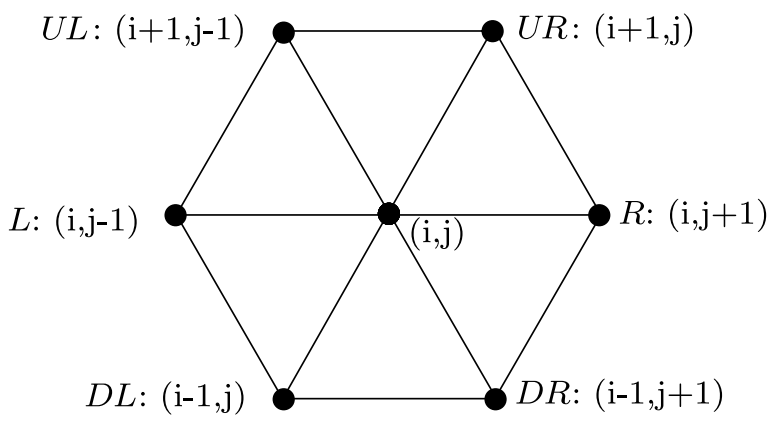


say Red, Green and Blue, coloring all the vertices of a hexagonal graph, such that each vertex colored with one of the three base colors, and no two adjacent vertices are of the same base color and similarly the same color. Furthermore, we assume a transitive order on these three base colors: namely, Red $<$ Green $<$ Blue.

\section{Multicoloring in Triangle-Free Hexagonal Graphs}

In this section, we shall study the problem of multicoloring a special type of hexagonal graph, say triangle-free hexagonal graph. In the next section, we will show that finding a good solution for this problem will lead to an algorithm for finding a good solution for a general hexagonal graph.

A graph is triangle-free if no three mutually-adjacent vertices have positive weights. For a given vertex $u$ with positive weight $w_{u}$, from this definition of trianglefree graph, only two possible configurations may exist for the structure of neighbors with positive weights, which are shown in Fig. 4. It is easy to see that if $u$ has 3 neighbors with positive weights, the neighboring vertices are of the same color. On the other hand, if the neighbors are of different colors, $u$ has at most 2 neighbors with positive weights. There exists a simple structure in triangle-free graph, i.e., a vertex has only one neighbor, we can regard this structure as the case in Fig. 4(b).

Consider vertex $u$ with positive weight $w_{u}$. Compute $c_{u}=w_{u}+\max \left\{w_{v} \mid v\right.$ is $u$ 's neighbor $\}. c_{u}$ is the weight of the maximum 2-clique adjacent to $u$, which also gives the minimum number of colors needed for multicoloring a triangle-free hexagonal graph. From the definition of $c_{u}$, any feasible coloring of vertex $u$ and its neighbors requires at least $c_{u}$ colors.

Let $d_{u}=\left\lceil c_{u} / 3\right\rceil$. For each vertex $u$, define four color sets, each of size $d_{u}$ :

1. $\operatorname{colorset}_{u}(\mathrm{Red})=\left\{j \in\left\{1, \ldots, 4 d_{u}\right\} \mid j=1 \bmod 4\right\}$,

2. $\operatorname{colorset}_{u}($ Green $)=\left\{j \in\left\{1, \ldots, 4 d_{u}\right\} \mid j=2 \bmod 4\right\}$,

3. $\operatorname{colorset}_{u}($ Blue $)=\left\{j \in\left\{1, \ldots, 4 d_{u}\right\} \mid j=3 \bmod 4\right\}$, and

4. $\operatorname{extraset}_{u}=\left\{j \in\left\{1, \ldots, 4 d_{u}\right\} \mid j=0 \bmod 4\right\}$.
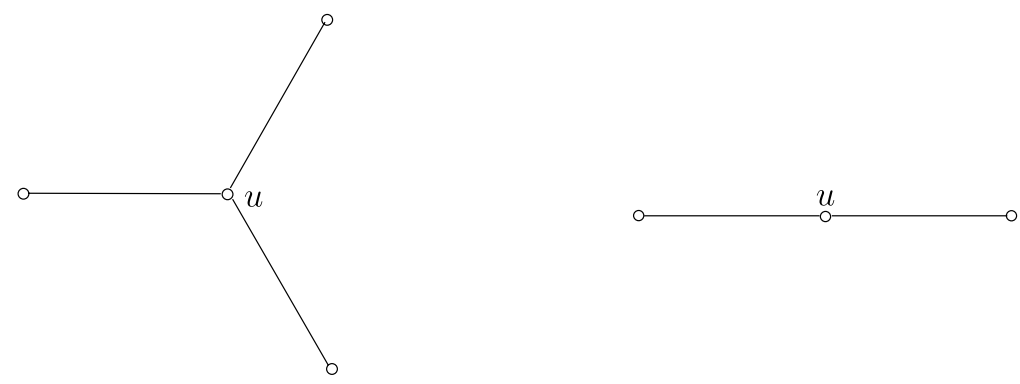

(a) Structure A: neighbors with the same base color

Fig. 4 Structure of neighbors with positive weights 
We will give a strategy to multicolor any vertex $u$ with weight $w_{u}$ by assigning $w_{u}$ colors from the above four sets so that no adjacent vertices are assigned the same color. The assignment strategy assigns multicolors to $u$ according to its base color and neighboring structure, which can be described as follows.

Assume vertex $u$ with base color $X$ has neighboring structure $A$, i.e. all its neighbors are of the same base color $Y \neq X$. Let the third base color be $Z$ where $Z \neq X$ and $Z \neq Y$. In this case, our strategy assigns multicolors to vertex $u$ first ${\text { from } \text { colorset }_{u}(X) \text {, then colorset }}_{u}(Z)$ if $\operatorname{colorset}_{u}(X)$ is not large enough, and finally colorset $_{u}(Y)$ if both $\operatorname{colorset}_{u}(X)$ and $\operatorname{colorset}_{u}(Z)$ are still not large enough.

On the other hand, if vertex $u$ with base color $X$ has neighboring structure $B$, then all three base colors appear in $u$ and its neighbors. The strategy first assigns multicolors from colorset $_{u}(X)$, and if not large enough, then from the extra color set extraset $_{u}$ and finally from $\operatorname{colorset}_{u}(Y)$ where base color $Y>Z \neq X$.

Note that in both cases, the colors in each color set may be assigned either from bottom to top or from top to bottom (i.e., from the lowest integer to the highest or from the highest to the lowest), depending on the base color or the parity of the vertex so as to avoid conflicts.

\section{The Strategy}

1. If vertex $u$ has no neighbors, just assign $w_{u}$ colors from 1 to $w_{u}$.

2. If vertex $u$ with base color $X$ has neighboring structure $A$ (Fig. 4(a)), let $Y$ be the base color of $u$ 's neighbors and $Z$ be the other third color. Assign $w_{u}$ multicolors to vertex $u$ as follows:

(a) Assign colors from colorset $(X)$ in bottom-to-top order.

(b) If not enough, assign colors from $\operatorname{colorset}_{u}(Z)$ in bottom-to-top order if $X<Y$; top-to-bottom otherwise.

(c) If still not enough, assign colors from $\operatorname{colorset}_{u}(Y)$ in top-to-bottom order.

3. If vertex $u$ with base color $X$ has neighboring structure $B$ (Fig. 4(b)), let $Y$ and $Z$ be the base colors of the left neighbor and the right neighbor, respectively. Without loss of generality, assume $Y>Z$. Assign $w_{u}$ multicolors to vertex $u$ as follows:

(a) Assign colors from colorset ${ }_{u}(X)$ in bottom-to-top order.

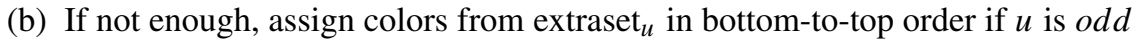
with respect to its two neighbors; top-to-bottom order otherwise.

(c) If still not enough, assign colors from $\operatorname{colorset}_{u}(Y)$ in top-to-bottom order.

Theorem 2 The above strategy is 1-local and can solve the multicoloring problem in triangle-free hexagonal graphs with an asymptotic approximation ratio 4/3.

Proof From the description of the strategy, it is clear that the colors assigned to any vertex depend only on neighboring information within distance 1, and thus, the strategy is 1-local.

To prove that the above strategy solves the multicoloring problem, we must prove that the colors assigned to any two adjacent vertices $u$ and $v$ are all different. As it turns out, we need to analyze the three cases shown in Fig. 5. $X$ and $Y$ denote the two respective different base colors of $u$ and $v$. It is easy to see that different kinds of color sets of $u$ and $v$ have no common colors. For example, $\operatorname{colorset}_{u}(\operatorname{Red}) \cap \operatorname{extraset}_{v}=\emptyset$. 


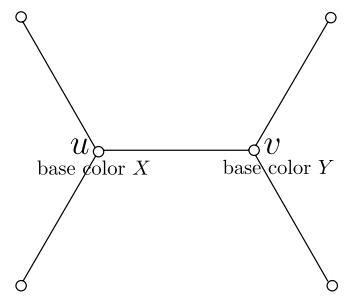

(a) Case $A$

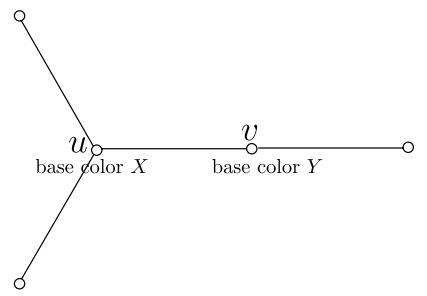

(b) Case $B$

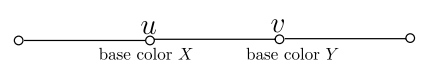

(c) Case $C$

Fig. 5 The local structure of vertices $u$ and $v$

From the definition of $c_{u}$, we have $c_{u} \geq w_{u}+w_{v}$ and, since $d_{u}=\left\lceil c_{u} / 3\right\rceil, c_{u} \leq 3 d_{u}$.

For Case A, the strategy would assign colors to $u$ from $\operatorname{colorset}_{u}(X)$, then

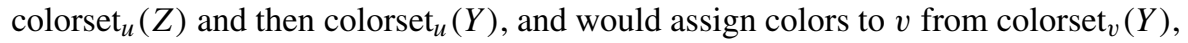
then $\operatorname{colorset}_{v}(Z)$ and then $\operatorname{colorset}_{v}(X)$. If the assigned colors of $u$ and $v$ are from different color sets, there is no confliction. Otherwise, $u$ and $v$ use colors from the same color set, $\operatorname{colorset}(X)=\operatorname{colorset}_{u}(X) \cup \operatorname{colorset}_{v}(X), \operatorname{colorset}(Y)=$ $\operatorname{colorset}_{u}(Y) \cup \operatorname{colorset}_{v}(Y)$ or $\operatorname{colorset}(Z)=\operatorname{colorset}_{u}(Z) \cup \operatorname{colorset}_{v}(Z)$. We study these three cases in the following:

(A-1) where $u$ is assigned colors from $\operatorname{colorset}_{u}(X)$ and $v$, after exhausting all colors in $\operatorname{colorset}_{v}(Y)$ and $\operatorname{colorset}_{v}(Z)$, is assigned colors from $\operatorname{colorset}_{v}(X)$. This means the weight $w_{v}$ is very large. Since $w_{u}+w_{v} \leq c_{u} \leq 3 d_{u}, w_{u}+w_{v} \leq$ $c_{v} \leq 3 d_{v}$, and since $u$ and $v$ use $\operatorname{colorset}(X)=\operatorname{colorset}_{u}(X) \cup \operatorname{colorset}_{v}(X)$ from opposite directions, $u$ and $v$ will not be assigned the same color;

(A-2) where $u$ is assigned colors from $\operatorname{colorset}_{u}(Z)$ and $v$ is assigned colors from $\operatorname{colorset}_{v}(Z)$. Then, all the colors in $\operatorname{colorset}_{u}(X)$ are assigned to $u$ and all the colors in $\operatorname{colorset}_{v}(Y)$ are assigned to $v$. Since $w_{u}+w_{v} \leq \min \left\{3 d_{u}, 3 d_{v}\right\}$, and since $u$ and $v$ use $\operatorname{colorset}(Z)=\operatorname{colorset}_{u}(Z) \cup \operatorname{colorset}_{v}(Z)$ from opposite directions (depending on whether $X<Y$ or otherwise), $u$ and $v$ will not be assigned the same color;

(A-3) where $u$ is assigned colors from $\operatorname{colorset}_{u}(Y)$ and $v$ is assigned colors from $\operatorname{colorset}_{v}(Y)$. By similar analysis as in case (A-1), we can say that $u$ and $v$ will not be assigned the same color.

In Case $\mathrm{B}$, the strategy would assign colors to $u$ from $\operatorname{colorset}_{u}(X)$, then $\operatorname{colorset}_{u}(Z)$ and then $\operatorname{colorset}_{u}(Y)$. Also, the strategy would assign colors to $v$

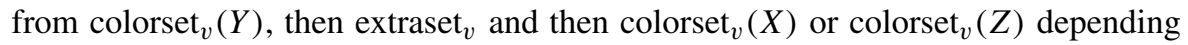
whether $X<Z$ or otherwise. If the assigned colors of $u$ and $v$ are from different color sets, there is no confliction. Otherwise, there are two subcases to be considered:

(B-1) where $u$ is assigned colors from $\operatorname{colorset}_{u}(X)$ and $v$ is assigned colors from $\operatorname{colorset}_{v}(X)$ after exhausting all colors in $\operatorname{colorset}_{v}(Y)$ and $\operatorname{extraset}_{v}$. This means the weight $w_{v}$ of $v$ is very large. Since $w_{u}+w_{v} \leq \min \left\{3 d_{u}, 3 d_{v}\right\}$, and since $u$ and $v$ use colorset $(X)=\operatorname{colorset}_{u}(X) \cup \operatorname{colorset}_{v}(X)$ from opposite directions, $u$ and $v$ will not be assigned the same color; 
(B-2) where $u$ is assigned colors from $\operatorname{colorset}_{u}(Y)$ and $v$ is assigned colors from $\operatorname{colorset}_{v}(Y)$. By similar analysis as in case (B-1), we can say that $u$ and $v$ will not be assigned the same color.

In Case $\mathrm{C}$, the strategy would assign colors to $u$ from $\operatorname{colorset}_{u}(X)$, then $\operatorname{extraset}_{u}$ and then $\operatorname{colorset}_{u}(Y)$ or $\operatorname{colorset}_{u}(Z)$, and would assign colors to $v$ from

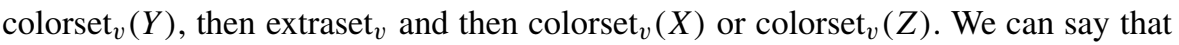
$u$ and $v$ cannot use colors from $\operatorname{colorset}_{u}(Z)$ and $\operatorname{colorset}_{v}(Z)$ at the same time. If it happens, the colors in $\operatorname{colorset}_{u}(X), \operatorname{colorset}_{v}(Y)$, extraset and extraset $_{v}$ are all used up, the total number of colors in these three color sets are no less than $3 \min \left\{d_{u}, d_{v}\right\}$, which is a contradiction to the $c_{u} \leq 3 d_{u}$ or $c_{v} \leq 3 d_{v}$. So we have to consider three subcases:

(C-1) where $u$ is assigned colors from $\operatorname{colorset}_{u}(X)$ and $v$ is assigned colors from $\operatorname{colorset}_{v}(X)$ after exhausting all colors in $\operatorname{colorset}_{v}(Y)$ and $\operatorname{extraset}_{v}$. This means the weight $w_{v}$ is very large. Since $w_{u}+w_{v} \leq \min \left\{3 d_{u}, 3 d_{v}\right\}$, and since $u$ and $v$ use $\operatorname{colorset}(X)=\operatorname{colorset}_{u}(X) \cup \operatorname{colorset}_{v}(X)$ from opposite directions, $u$ and $v$ will not be assigned the same color;

(C-2) where $u$ is assigned colors from $\operatorname{extraset}_{u}$ and $v$ is assigned colors from extraset $_{v}$. This means all the colors in $\operatorname{colorset}_{u}(X)$ and $\operatorname{colorset}_{v}(Y)$ have been assigned to $u$ and $v$, respectively. Since $w_{u}+w_{v} \leq \min \left\{3 d_{u}, 3 d_{v}\right\}$, and since $u$ and $v$ use extraset $=$ extraset $_{u} \cup$ extraset $_{v}$ from opposite directions (the parities of $u$ and $v$ are different), $u$ and $v$ will not be assigned the same color;

(C-3) where $u$ is assigned colors from $\operatorname{colorset}_{u}(Y)$ and $v$ is assigned colors from $\operatorname{colorset}_{v}(Y)$. By similar analysis as in case $(\mathrm{C}-1)$, we can say that $u$ and $v$ will not be assigned the same color.

For the whole triangle-free hexagonal graph, the maximal weight clique (2-clique) $c=\max _{u}\left\{c_{u}\right\}$ is a lower bound on the optimal value, and our algorithm uses at most $4 \max _{u}\left\{\left\lceil c_{u} / 3\right\rceil\right\}$ colors. Thus, the above strategy has an asymptotic approximation ratio of $4 / 3$.

From Lemma 1, we can easily have a 1-local asymptotic 4/3-competitive online algorithm for frequency allocation in triangle-free cellular networks.

\section{Multicoloring in Hexagonal Graphs}

In this section, we consider multicoloring hexagonal graphs. Our strategy works in two stages. In the first stage, each vertex assigns colors using local information on the weights of this vertex and its neighboring vertices. After the first stage, some vertices may be unsatisfied, i.e. not all of the necessary colors have been assigned, and the unsatisfied vertices, along with the edges connecting them, form a triangle-free graph. Applying the algorithm in the previous section, each vertex can be assigned colors, to satisfy all the remaining unsatisfied vertices, by using 1-local information. Combining these two stages, we have a 1-local algorithm for multicoloring hexagonal graphs. 
We now describe the first stage, which is similar to the first stage in [12]. In [12], the algorithm needs to have the global information about the maximum weights of "all" 3-cliques in the graph (stage 1) so as to have an acyclic graph of the remaining unsatisfied vertices (for stage 2). As for an algorithm which is 1-local, only the maximal weights of the local 3-cliques will be available (stage 1) and a triangle-free hexagonal graph (which can be cyclic) will result (for stage 2). Consider vertex $u$ with base color $X$. Let $C_{u}$ be the maximal weight among the 3-cliques including $u$, and let $k_{u}=\left\lceil C_{u} / 3\right\rceil$. For the three base colors Red, Green and Blue, we define a cyclic order among them as Red $\rightarrow$ Green, Green $\rightarrow$ Blue and Blue $\rightarrow$ Red. If $X \rightarrow Y$, let $m_{u}$ be the maximal weight of the neighboring vertices with color $Y$. We define color sets: $\operatorname{colorset}_{u}(\operatorname{Red})=\left\{j \in\left\{1, \ldots, 3 k_{u}\right\} \mid j \equiv 1 \bmod 3\right\}, \operatorname{colorset}_{u}($ Green $)=$ $\left\{j \in\left\{1, \ldots, 3 k_{u}\right\} \mid j \equiv 2 \bmod 3\right\}$ and $\operatorname{colorset}_{u}($ Blue $)=\left\{j \in\left\{1, \ldots, 3 k_{u}\right\} \mid j \equiv\right.$ $0 \bmod 3\}$. In the first stage, vertex $u$ with base color $X$ and weight $w_{u}$ is assigned colors from these sets using the strategy described as follows:

1. Vertex $u$ is assigned colors from $\operatorname{colorset}_{u}(X)$ in bottom-to-top order.

2. If not enough and $m_{u}<k_{u}$, vertex $u$ is assigned the upper $\min \left\{k_{u}-m_{u}, w_{u}-k_{u}\right\}$ colors from $\operatorname{colorset}_{u}(Y)$.

After the first stage, each vertex has been assigned with some colors. The remaining graph contains only those vertices whose colors have not been totally satisfied, i.e., the number of assigned colors in vertex $u$ is less than its weight.

Lemma 3 The remaining graph is triangle-free, i.e., contains no 3-clique.

Proof If some vertex $u$ is still unsatisfied, it must be that $w_{u}>\max \left\{k_{u}, 2 k_{u}-m_{u}\right\}$. Thus, the remaining unsatisfied weight in vertex $u$ is $w_{u}^{\prime}=w_{u}-\max \left\{k_{u}, 2 k_{u}-m_{u}\right\}$. For any three mutually-adjacent vertices (3-clique) $u, v$ and $t, \operatorname{since} \min \left\{C_{u}, C_{v}, C_{t}\right\} \geq$ $w_{u}+w_{v}+w_{t} \geq 3 \min \left\{w_{u}, w_{v}, w_{t}\right\}$, we have $\min \left\{k_{u}, k_{v}, k_{t}\right\} \geq \min \left\{w_{u}, w_{v}, w_{t}\right\}$. Without loss of generality, assume $w_{u}=\min \left\{w_{u}, w_{v}, w_{t}\right\}$, then obviously $k_{u} \geq w_{u}$ and $w_{u}^{\prime}=0$. From the description of coloring strategy, we can say that at most two of $\left\{w_{u}^{\prime}, w_{v}^{\prime}, w_{t}^{\prime}\right\}$ are strictly positive, which means at least one of the vertices $(u, v$ and $t)$ has all its required colors totally assigned in the first stage. Therefore, the remaining graph contains no 3-clique, i.e., is a triangle-free hexagonal graph.

Lemma 4 The total weight of two neighboring vertices $u$ and $v$ in the remaining graph is at most $\left\lfloor\min \left\{C_{u}, C_{v}\right\} / 3\right\rfloor$.

Proof For the remaining unsatisfied vertices, since $w_{u}^{\prime}=w_{u}-\max \left\{k_{u}, 2 k_{u}-m_{u}\right\}$, we have $w_{u}^{\prime} \leq w_{u}-\left(2 k_{u}-m_{u}\right)=w_{u}+m_{u}-2 k_{u} \leq C_{u}-2 k_{u}$. For any two adjacent unsatisfied vertices $u$ and $v$, we can also get $w_{u}^{\prime}+w_{v}^{\prime} \leq w_{u}-k_{u}+w_{v}-k_{v}$. If $C_{u} \geq C_{v}$, which implies $k_{u} \geq k_{v}$, then we have $w_{u}^{\prime}+w_{v}^{\prime} \leq C_{v}-2 k_{v} \leq\left\lfloor C_{v} / 3\right\rfloor$ as $C_{v} \geq w_{u}+w_{v}$. Similarly, if $C_{u} \leq C_{v}$, which implies $k_{u} \leq k_{v}$, then we have $w_{u}^{\prime}+w_{v}^{\prime} \leq$ $C_{u}-2 k_{u} \leq\left\lfloor C_{u} / 3\right\rfloor$. Thus, the total remaining weight of any two adjacent unsatisfied vertices is at most $\min \left\{\left\lfloor C_{u} / 3\right\rfloor,\left\lfloor C_{v} / 3\right\rfloor\right\}$.

From Lemma 3, the remaining graph is triangle-free, so in the second stage, we can use the algorithm in Sect. 3 to process the remaining unsatisfied vertices. Each 
vertex gets the remaining weight information from its adjacent vertices and the total number of colors used in this stage is at most $4 \max _{u}\left\{\left\lceil\left\lfloor C_{u} / 3\right\rfloor / 3\right\rceil\right\}=4 \max _{u}\left\lceil\frac{C_{u}-2}{9}\right\rceil$ (Theorem 2 and Lemma 4).

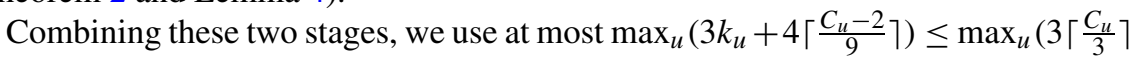
$\left.+4\left\lceil\frac{C_{u}-2}{9}\right\rceil\right) \leq \frac{13}{9} C_{u}+7$ colors. Since $C_{u}$ is a lower bound on the optimal solution, the asymptotic approximation ratio for our strategy is $13 / 9$.

Thus, we have the following theorem.

Theorem 5 There exists a 1-local asymptotic 13/9-competitive algorithm for the multicoloring problem in hexagonal graphs.

\section{Conclusion}

We have given an asymptotic 13/9-approximation algorithm for multicoloring hexagonal graphs. This implies an asymptotic 13/9-competitive solution for the online frequency allocation problem, which involves servicing calls in each cell in a cellular network. The distributed algorithm is practical in the sense that frequency allocation can be done based on information about its neighbors and itself only. We note that, in fact, when calls are requested or released in a cell, a constant number of frequencies might have to be reassigned so as to actually achieve this asymptotic 13/9-competitive bound.

Acknowledgements The authors thank Dr. Bethany M.Y. Chan for her efforts in making this paper more readable.

\section{References}

1. Aardal, K.I., van Hoesel, S.P.M., Koster, A.M.C.A., Mannino, C., Sassano, A.: Models and solution techniques for frequency assignment problems. Q. J. Belg. Fr. Italian Oper. Res. Soc. (4OR) 1(4), 261-317 (2003)

2. Chan, W.-T., Chin, F.Y.L., Ye, D., Zhang, Y., Zhu, H.: Frequency allocation problem for linear cellular networks. In: Proceedings of the 17th Annual International Symposium on Algorithms and Computation (ISAAC 2006), Dec. 2006. Lecture Notes in Computer Science, vol. 4288, pp. 61-70. Springer, Berlin (2006)

3. Chan, W.-T., Chin, F.Y.L., Ye, D., Zhang, Y., Zhu, H.: Greedy online frequency allocation in cellular networks. Inf. Process. Lett. 102, 55-61 (2007)

4. Chan, W.-T., Chin, F.Y.L., Ye, D., Zhang, Y.: Online frequency allocation in cellular networks. In: Proceedings of the 19th ACM Symposium on Parallelism in Algorithms and Architectures (SPAA 2007), pp. 241-249

5. Caragiannis, I., Kaklamanis, C., Papaioannou, E.: Efficient on-line frequency allocation and call control in cellular networks. Theory Comput. Syst. 35(5), 521-543 (2002)

6. Hale, W.: Frequency assignment: theory and applications. Proc. IEEE 68(12), 1497-1514 (1980)

7. Havet, F.: Channel assignment and multicoloring of the induced subgraphs of the triangular lattice. Discrete Math. 233, 219-231 (2001)

8. Janssen, J., Krizanc, D., Narayanan, L., Shende, S.M.: Distributed online frequency assignment in cellular networks. J. Algorithms 36(2), 119-151 (2000)

9. Jaumard, B., Marcotte, O., Meyer, C.: Mathematical models and exact methods for channel assignment in cellular networks. In: Sansò, B., Soriano, P. (eds.) Telecommunications Network Planning, pp. 239-255. Kluwer, Amsterdam (1999) 
10. Katzela, I., Naghshineh, M.: Channel assignment schemes for cellular mobile telecommunication systems: a comprehensive survey. IEEE Pers. Commun. 3(3), 10-31 (1996)

11. MacDonald, V.: Advanced mobile phone service: the cellular concept. Bell Syst. Tech. J. 58(1) (1979)

12. McDiarmid, C., Reed, B.A.: Channel assignment and weighted coloring. Networks 36(2), 114-117 (2000)

13. Narayanan, L.: Channel assignment and graph multicoloring. In: Stojmenović, I. (ed.) Handbook of Wireless Networks and Mobile Computing, pp. 71-94. Wiley, New York (2002)

14. Narayanan, L., Shende, S.M.: Static frequency assignment in cellular networks. Algorithmica 29(3), 396-409 (2001)

15. Narayanan, L., Tang, Y.: Worst-case analysis of a dynamic channel assignment strategy. Discrete Appl. Math. 140(1-3), 115-141 (2004)

16. Sparl, P., Zerovnik, J.: 2-local 5/4-competitive algorithm for multicoloring triangle-free hexagonal graphs. Inf. Process. Lett. 90, 239-246 (2004)

17. Sparl, P., Zerovnik, J.: 2-local 4/3-competitive algorithm for multicoloring hexagonal graphs. J. Algorithms 55(1), 29-41 (2005) 